\title{
Hearing Loss in Mucopolysaccharidosis
}

\author{
Cibele Gomes Bicalho ${ }^{1}$ Emília Katiane Embiruçu de Araújo Leão ${ }^{2,3}$ Álvaro Muiños de Andrade ${ }^{1}$ \\ Angelina Xavier Acosta 2,4 \\ 1 Department of Otorhinolaryngology, Hospital Universitário \\ Professor Edgard Santos, Salvador, BA, Brazil \\ 2 Medical Genetics Service, Hospital Universitário Professor Edgard \\ Santos (HUPES), Salvador, BA, Brazil \\ ${ }^{3}$ Department of Life Sciences, Universidade Estadual da Bahia (UNEB), \\ BA, Brazil \\ ${ }^{4}$ Department of Pediatrics, Universidade Federal da Bahia, Salvador, \\ BA, Brazil \\ Address for correspondence Cibele Gomes Bicalho, Master, Clioc - \\ Clinica de Otorrinolaringologia, Av Anita Garibaldi 1555 sala 601, \\ Salvador, BA, 40170-130 (e-mail: cibelebicalho@gmail.com). \\ Int Arch Otorhinolaryngol 2021;25(3):e386-e391.
}

\section{Introduction}

Mucopolysaccharidosis (MPS) comprises several rare diseases caused by deficiencies in the lysosomal enzymes, leading to the accumulation of glycosaminoglycans (GAG) in organs and tissues that result in multisystemic, chronic, and progressive clinical manifestations. In all, 11 enzymatic deficiencies have been identified, corresponding to 7 different types of MPS. ${ }^{1,2}$

The incidence of MPS ranges from 3.4 to 4.5 in 100,000 live births. ${ }^{3}$ In Brazil, MPS type II is the most common variant, followed by MPS types I and VI. The frequency of MPS VI in Brazil is relatively high compared with other countries, particularly in the Brazilian North/Northeast. ${ }^{4}$

Each type of MPS is considered genetically, as well as clinically, heterogeneous. Clinical signs, often absent at birth, appear gradually, including delayed neuropsychomotor development, facial dysmorphism, skeletal dysplasia and hearing loss (HL), in addition to frequent respiratory and cardiovascular complications. ${ }^{2}$

Hearing loss is one of the main clinical otolaryngologic manifestations seen in association with MPS, present in received

December 13, 2019

accepted

March 16, 2020

published online

September 24, 2020
DOI https://doi.org/ 10.1055/s-0040-1712107. ISSN 1809-9777.

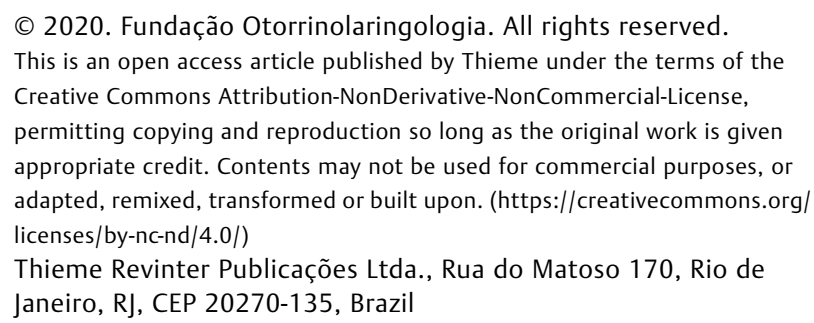


more than $80 \%$ of all cases. ${ }^{5}$ Not only does the literature contain scarce data on hearing impairment, butthe underlying process that causes these manifestations remains unclear. Consequently, this leaves health care professionals unsure of how to effectively treat these conditions in MPS patients.

The treatment of MPS, initially symptomatic and palliative in nature, is performed by multidisciplinary teams, involving the participation of diverse medical specialists, including cardiologists, pulmonologists, anesthesiologists, orthopedic and ENT specialists, ophthalmologists and neurosurgeons, among others, as well as physical, occupational, and speech therapists, in addition to psychologists. ${ }^{6}$ Currently, enzyme replacement therapy (ERT) is also available for MPS types I, II, $\mathrm{IV}$, and VI, involving periodic intravenous administration of specific deficient enzymes. This treatment has provided encouraging results by improving pulmonary function, decreasing urinary excretion of GAG and reducing hepatomegaly, as well as improving sleep apnea. ${ }^{7,8}$

The present study aimed to clarify the natural evolution of hearing impairment in MPS patients and provide relevant information in an effort to enhance therapeutic intervention.

\section{Methods}

A case series was evaluated to ascertain the descriptive data related to hearing loss in the MPS patients monitored at an MPS reference service located in Brazil. The time period ranged from the first otorhinolaryngologic and audiological evaluation described in each patient's medical records to the prospective ear-nose-throat (ENT) and audiological followup these patients received at the service between December 2012 and October 2014.

Patients were included if they were diagnosed with MPS, agreed to participate in the study, provided informed written consent and had a prior clinical ENT evaluation, as well as at least one additional audiological examination.

The medical records of 24 MPS patients were reviewed, specifically pertaining to data regarding previously performed clinical ENT and audiological evaluations. Each patient's initial clinical ENT evaluation was identified in their medical records, and, if none was found, an initial exam was performed by an ENT physician. All patients were followed from December 2012 to October 2014, with ENT, audiometry and/or auditory brainstem response(ABR) and/or otoacoustic emission (OAE) exams performed at least 6 months after the initial assessment. The following data were collected for analysis: age, MPS type, duration of ERT, age at time of ERT onset, otologic signs and symptoms, and audiological evaluation results. None of these examinations posed any risk to patients, as all are routine audiological assessments commonly performed in patients with MPS. In the cases of 15 out of the 24 patients who presented both initial and follow-up ENT and audiological assessments (see - Table 1), these were submitted for data analysis.

No patients underwent any surgical procedures.

Audiological evaluations were performed by tonal audiometry to classify the degree of HL in accordance with the criteria established by Silman and Silverman (1997). The tonal averages of 500, 1,000 and 2,000 kHz were measured, as previously determined by Lloyd and Kaplan (1978) for patients aged 7 years or older. The classification established by Northern and Downs (1984) was used for children younger than 7 years old. Additionally, pure tone audiometry, impedance audiometry, and evoked potential ABR) tests were performed.

Despite limitations inhibiting direct comparisons among groups, descriptive statistics (proportions, central tendency measures and dispersion) were calculated with respect to relevant variables whenever possible. Statistical analysis was performed using available data from audiometry testing and/or patient records. The degree of HL was established using data from the patient's best ear and was considered to be moderate when classified as either moderate or moderately severe.

The present study received approval from the hospital's institutional review board, protocol number 99/10.

\section{Results}

Among the 24 patients who presented or received an initial ENT evaluation, the age ranged from 1 to 21 years (mean: 7.3 years). Fifteen patients (62.5\%) were female and 20 (83\%) had been on ERT for 0 to 84 months (mean: 19.9 months). Among those who had not undergone ERT, two had MPS type II, one had MPS type IIIA, and one had MPS type IVA.

Of these 24 patients, 15 successfully completed follow-up hearing assessment and audiological testing, thus permitting comparative data analysis in these cases. All of these patients had been on ERT for periods ranging from 1.7to 14.5 years (mean: 5 years).

At the time of initial ENT evaluation, $37.5 \%$ of the 24 patients reported hypoacusis, and $37.5 \%$ had a history of otitis. Among the patients suffering from hearing impairment, around 30\% noted a progressive worsening in HL. - Table 2 shows the frequency of hypoacusis by MPS type at the time of initial and most recent ENT evaluations. No patients had used hearing aids, nor had any undergone ventilation tube insertion procedures. Only one patient received a referral for ventilation tube surgery during the study period. At the time of the followup ENT evaluation, complaints of HL were registered by $45.4 \%$ of the 15 patients, $80 \%$ of whom reported stability in terms of hearing impairment progression. Approximately 20\% of the patients reported otitis during the year prior to follow-up assessment.

Otoscopy findings from the initial ENT evaluations were normal in 8 of the 24 patients (33.3\%), while 1 patient (4\%) presented tympanic membrane(TM) perforation. Tympanic membrane retraction was seen bilaterally in 7 patients (29.1\%) and unilaterally in 2 (8.3\%). Among the patients with TM retraction, only two patients had otitis media with effusion (OME). At the time of their follow-up ENT evaluation, only 2 of 15 patients had a normal bilateral otoscopic assessment, the frequency of bilateral TM retraction had risen to $60 \%$, and individual otoscopic assessment found a greater number of patients with otoscopic alterations (- Table 3). 
Table 1 Clinical data, ear-nose-throat, and audiological examinations from mucopolysaccharidosis patients

\begin{tabular}{|c|c|c|c|c|c|c|c|c|c|c|c|c|}
\hline $\mathrm{N}$ & $\begin{array}{l}\text { MPS } \\
\text { type }\end{array}$ & Age $^{*}$ & ERT* $^{*}$ & $\begin{array}{l}1^{\text {st }} \text { ENT } \\
\text { evaluation }\end{array}$ & $\begin{array}{l}\text { Tonal } \\
\text { audio } \\
(1)\end{array}$ & $\begin{array}{l}\text { Vocal } \\
\text { audio } \\
(1)\end{array}$ & $\begin{array}{l}\text { Tympanometry } \\
\text { (1) }\end{array}$ & $A B R$ & $\begin{array}{l}2^{\text {nd }} \text { ENT } \\
\text { evaluation }\end{array}$ & $\begin{array}{l}\text { Tonal } \\
\text { audio } \\
(2)\end{array}$ & $\begin{array}{l}\text { Vocalaudio } \\
\text { (2) }\end{array}$ & $\begin{array}{l}\text { Tympanometry } \\
\text { (2) }\end{array}$ \\
\hline 1 & $\mathrm{VI}$ & 2 & 4 & $x$ & NA & NA & $x$ & $\mathrm{NA}$ & $x$ & $\mathrm{NA}$ & $\mathrm{NA}$ & $x$ \\
\hline 2 & 1 & 8 & 27 & $x$ & $x$ & NA & $x$ & $\mathrm{NA}$ & NA & $\mathrm{NA}$ & NA & $\mathrm{NA}$ \\
\hline 3 & $\mathrm{VI}$ & 1 & 4 & $x$ & $x$ & $x$ & $x$ & $x$ & $x$ & $x$ & $x$ & $x$ \\
\hline 4 & 1 & 13 & 60 & $x$ & $x$ & NA & $x$ & $x$ & $x$ & $x$ & $x$ & $x$ \\
\hline 5 & $\mathrm{VI}$ & 14 & 18 & $x$ & $x$ & NA & $x$ & $\mathrm{NA}$ & $x$ & $x$ & $x$ & $x$ \\
\hline 6 & $\mathrm{VI}$ & 3 & 0 & $x$ & NA & $x$ & $x$ & NA & NA & NA & NA & NA \\
\hline 7 & $\mathrm{VI}$ & 9 & 6 & $x$ & $x$ & $x$ & $x$ & $\mathrm{NA}$ & $\mathrm{NA}$ & $\mathrm{NA}$ & NA & $\mathrm{NA}$ \\
\hline 8 & $\mathrm{VI}$ & 10 & 6 & $x$ & $x$ & NA & $x$ & NA & $x$ & $x$ & $x$ & $x$ \\
\hline 9 & II & 3 & 84 & $x$ & $x$ & $\mathrm{NA}$ & $x$ & $\mathrm{NA}$ & $\mathrm{NA}$ & $\mathrm{NA}$ & $\mathrm{NA}$ & $\mathrm{NA}$ \\
\hline 10 & $\mathrm{VI}$ & 5 & 18 & $x$ & $x$ & NA & $x$ & $x$ & $x$ & $x$ & $x$ & $x$ \\
\hline 11 & $\mathrm{VI}$ & 2 & 27 & $x$ & $x$ & $\mathrm{NA}$ & $x$ & $\mathrm{NA}$ & $x$ & $x$ & $x$ & $x$ \\
\hline 12 & $\mathrm{VI}$ & 3 & 0 & $x$ & $x$ & NA & $x$ & $x$ & $x$ & $x$ & $x$ & $x$ \\
\hline 13 & $\mathrm{VI}$ & 8 & 0 & $x$ & $x$ & $x$ & $x$ & $x$ & $\mathrm{NA}$ & $\mathrm{NA}$ & NA & $\mathrm{NA}$ \\
\hline 14 & $\mathrm{VI}$ & 12 & 24 & $x$ & $x$ & NA & $x$ & NA & $x$ & $x$ & NA & $x$ \\
\hline 15 & $\mathrm{VI}$ & 8 & 27 & $x$ & $x$ & $x$ & $x$ & NA & $x$ & $x$ & $x$ & $x$ \\
\hline 16 & $\mathrm{VI}$ & 8 & 30 & $x$ & $x$ & $x$ & $x$ & $x$ & $x$ & $x$ & $x$ & $x$ \\
\hline 17 & $\mathrm{VI}$ & 2 & 20 & $x$ & NA & NA & $x$ & NA & $x$ & $x$ & $x$ & $x$ \\
\hline 18 & II & 4 & 24 & $x$ & $x$ & $x$ & $x$ & $x$ & $x$ & $x$ & $x$ & $x$ \\
\hline 19 & II & 10 & 16 & $x$ & $x$ & $x$ & $x$ & NA & $x$ & $x$ & $x$ & $x$ \\
\hline 20 & II & 21 & 84 & $x$ & $x$ & NA & $x$ & $x$ & $x$ & $x$ & $x$ & $x$ \\
\hline 21 & II & 4 & 0 & $x$ & NA & $x$ & $x$ & $x$ & NA & NA & NA & NA \\
\hline 22 & IVA & 11 & 0 & $x$ & $x$ & NA & $x$ & $\mathrm{NA}$ & $\mathrm{NA}$ & $\mathrm{NA}$ & NA & NA \\
\hline 23 & IIIA & 7 & 0 & $x$ & NA & NA & $x$ & NA & NA & NA & NA & NA \\
\hline 24 & II & 8 & 0 & $x$ & NA & NA & $x$ & NA & NA & NA & NA & NA \\
\hline
\end{tabular}

Abbreviations: ABR, auditory brainstem response;ENT, ear-nose-throat; ERT, enzyme-replacement therapy; MPS, mucopolysaccharidosis; NA, not available; $X$, available.

Initial audiometric evaluations were found to have occurred when patients had been on ERT for 0 to 8 years (mean: 2.3 years). Among the 24 audiometric results considered, 18 patients had undergone testing procedures providing pure tone and/or vocal audiometry responses. One patient who had an unsatisfactory response under pure tone audiometry was submitted to speech audiometry for HL assessment. The patients with pure tone audiometry

Table 2 Frequency of hypoacusis in accordance with mucopolysaccharidosistype

\begin{tabular}{|l|l|l|}
\hline MPS type & $\begin{array}{l}\text { Hypoacusis (n/ N/ \%) } \\
\text { Initial ENT evaluation }\end{array}$ & $\begin{array}{l}\text { Hypoacusis (n/ N/ \%) } \\
\text { Follow-up ENT } \\
\text { evaluation }\end{array}$ \\
\hline $\mathrm{I}$ & $1 / 2(50 \%)$ & $1 / 1(100 \%)$ \\
\hline $\mathrm{II}$ & $4 / 6(66.6 \%)$ & $3 / 3(100 \%)$ \\
\hline $\mathrm{III}-\mathrm{A}$ & $0 / 1$ & NA \\
\hline $\mathrm{IV}-\mathrm{A}$ & $0 / 1$ & $\mathrm{NA}$ \\
\hline $\mathrm{VI}$ & $4 / 14(28.6 \%)$ & $5 / 11(45.4 \%)$ \\
\hline Total & $9 / 24(37.5 \%)$ & $9 / 15(60 \%)$ \\
\hline
\end{tabular}

Abbreviations: ENT, ear-nose-throat; MPS, mucopolysaccharidosis. results were aged between 5 and 16 years (mean: 10 years). Hearing loss was detected in 17 (94.4\%) of these patients. Among those who presented tonal audiometry findings, 9 (56.2\%) had bilateral conductive hearing loss, 1 (6.2\%) had unilateral conductive hearing loss, 2 (12\%) had mixed bilateral hearing loss, 2 (12\%) had bilateral sensorineural loss, and the remaining 2 presented unilateral conductive hearing loss associated with mixed $\mathrm{HL}$ and sensorineural loss, respectively. With respect to degree of HL,75\% of 18 patients were classified as mild, $18.8 \%$ had moderate,and only 1 patient presented severe hearing loss. Tympanometry findings were available for 23 patients and type $B$ curve was present in $50 \%$, with $68.3 \%$ presenting a bilateral type-B curve.

Of the 17 patients with HL, 6 (35.2\%) reported complaints of hypoacusis.

One patient presented no evidence of HL upon audiological evaluation, yet reported experiencing hypoacusis.

Follow-up audiometric assessments were obtained for 15 patients, 14 of whom presentedan audiometric tonal response. The ages of the patients ranged between 5 and 20 years (mean: 12 years), and HL was detected in 13 (92.9\%) of these patients. 
Table 3 Frequency of otoscopic findings

\begin{tabular}{|l|l|l|l|l|}
\hline & \multicolumn{2}{|l|}{ Initial ENT evaluation(n/ N/ \%) } & \multicolumn{2}{l|}{ Follow-up ENT evaluation(n/ N/ \%) } \\
\hline Otoscopy & RE & LE & RE & LE \\
\hline OME & $1 / 24(4 \%)$ & $2 / 24(8 \%)$ & $1 / 15(6 \%)$ & $1 / 15(6 \%)$ \\
\hline TM Opacification & $3 / 24(12 \%)$ & $3 / 24(12 \%)$ & $0 / 15$ & $1 / 15(6 \%)$ \\
\hline TM Perforation & $2 / 24(9 \%)$ & $1 / 24(4 \%)$ & $0 / 15$ & $0 / 15$ \\
\hline TM Retraction & $8 / 24(33 \%)$ & $8 / 24(33 \%)$ & $10 / 15(67)$ & $11 / 15(73 \%)$ \\
\hline Normal & $8 / 24(33 \%)$ & $9 / 24(37 \%)$ & $4 / 15(27 \%)$ & $3 / 15(20 \%)$ \\
\hline
\end{tabular}

Abbreviations: ENT, ear-nose-throat; LE, left ear; OME, otitis media with effusion; RE, right ear; TM, tympanic membrane.

Comparisons between initial and follow-up audiometric exams revealed that one patient exhibited normal auditory thresholds, while the sensorineural component developed in two patients. One patient with an initial assessment of sensorineural HL later presented mixed HL on a later audiometric assessment. All 10 patients who initially had bilateral conductive HL or bilateral mixed HL continued to experience the same type of impairment upon later examination.

With respect to the degree of $\mathrm{HL}, 85 \%$ of the patients with mild conductive HL demonstrated worsening over time that evolved to a moderate classification upon final ENT assess- ment. One patient exhibited improvement with respect to degree of HL.

Nine of the 24 patients with an initial ABR examination were aged 5 to 17 years (average: 12 years). Electrophysiological threshold testing was performed in 6 patients, ranging from 35 to $90 \mathrm{~dB}$. Nerve conduction was evaluated in 8 patients, with normal readings obtained in $88 \%$ of the cases. One patient presented latency in all waves with normal interpeaks. Seven (77.7\%) patients who underwent ABR presented conductive $\mathrm{HL}$ on pure tone audiometry. - Table 4 shows the ABR findings with respect to MPS type.

Table 4 Pure tone audiometry and ABR data (obtained from mucopolysaccharidosispatient records)

\begin{tabular}{|c|c|c|c|c|c|c|c|c|c|}
\hline $\begin{array}{l}\text { MPS } \\
\text { type }\end{array}$ & Age & ERT & $\begin{array}{l}\text { Type of hearing } \\
\text { impairment }\end{array}$ & $\begin{array}{l}\text { Degree of } \\
\text { hearing loss }\end{array}$ & $\begin{array}{l}\text { ABR } \\
\text { (threshold) }\end{array}$ & Age & ERT & $\begin{array}{l}\text { Type of hearing } \\
\text { impairment }\end{array}$ & $\begin{array}{l}\text { Degree of } \\
\text { hearing loss }\end{array}$ \\
\hline I & 13 & 56 & C/Normal & Normal & NA & & & NA & NA \\
\hline I & 7 & 24 & Normal & Normal & NA & 17 & 103 & Normal & Normal \\
\hline II & 13 & 84 & Mixed/Mixed & Mild & & & & NA & $\mathrm{NA}$ \\
\hline II & 14 & 25 & Mixed/Mixed & Severe & $90 / 80$ & 17 & 64 & Mixed/Mixed & Severe \\
\hline II & 10 & 16 & $\mathrm{C} / \mathrm{SN}$ & Mild & NA & 10 & 25 & SN/SN & At higher frequencies \\
\hline II & 16 & 24 & SN/SN & Mild & NA & 20 & 60 & SN/SN & Moderate \\
\hline II & 13 & 0 & NA & NA & $60 / 60$ & & & NA & NA \\
\hline IVA & 10 & 0 & $C / C$ & Moderate & & & & & \\
\hline $\mathrm{VI}$ & 13 & 9 & $\mathrm{C} / \mathrm{C}$ & Mild & NA & 17 & 32 & $C / C$ & Moderate \\
\hline $\mathrm{VI}$ & 11 & 26 & $C / C$ & Mild & $\mathrm{NA}$ & & & & \\
\hline $\mathrm{VI}$ & 9 & 6 & $C / C$ & Mild & NA & 12 & 30 & C/ Mixed & Moderate \\
\hline $\mathrm{VI}$ & 5 & 32 & $\mathrm{C} / \mathrm{C}$ & Mild & $40 / 40$ & 9 & 64 & $\mathrm{C} / \mathrm{C}$ & Moderate \\
\hline $\mathrm{VI}$ & 10 & 96 & C/Mixed & Moderate & NA & 13 & 132 & $\mathrm{C} / \mathrm{C}$ & Mild \\
\hline $\mathrm{VI}$ & 6 & 36 & $C / C$ & Mild & $50 / 50$ & 10 & 80 & $C / C$ & Moderate \\
\hline $\mathrm{VI}$ & 10 & 26 & $C / C$ & Mild & $35 / 35$ & & & & \\
\hline $\mathrm{VI}$ & 10 & 20 & $C / C$ & Mild & $\mathrm{NA}$ & 11 & 36 & $C / C$ & Moderate \\
\hline $\mathrm{VI}$ & 8 & 28 & SN/SN & Moderate & NA & 12 & 71 & Mixed/Mixed & Moderate \\
\hline $\mathrm{VI}$ & 9 & 29 & $\mathrm{C} / \mathrm{C}$ & Mild & $45 / 45$ & 12 & 74 & $\mathrm{C} / \mathrm{C}$ & Mild \\
\hline $\mathrm{VI}$ & 5 & 68 & $C / C$ & Mild & $\mathrm{NA}$ & & & & \\
\hline
\end{tabular}

Abbreviations: ABR, auditory brainstem response; C, conductive; ERT, enzyme-replacement therapy; MPS, mucopolysaccharidosis; NA, Not available; SN, sensorineural. 
All data pertaining to audiometry, ABR, and ERT are listed in - Table 4.

\section{Discussion}

Regrettably, the true prevalence of hearing impairment in patients with MPS remains unknown. These individuals frequently present neurological damage, which poses a considerable challenge to conducting satisfactory audiometric evaluations. Nonetheless, hypoacusis is a common finding and, in the present sample, $42.3 \%$ of these patients and/or their parents/legal guardians reported the presence of HL. Interestingly, just $71.4 \%$ of patients with MPS type II reported hearing impairment, while the audiometry testing detected HL in $94.4 \%$ of all the MPS patients considered herein. This finding is in agreement with a previous study published in 2012, in which $67.5 \%$ of patients with MPS type II reported HL and, following audiometry, $94 \%$ were found to have hearing impairment loss. ${ }^{9}$ Meanwhile, other studies have demonstrated a similarly elevated prevalence of HL in patients with MPS. ${ }^{5,10}$

Approximately 11 (60\%) patients who denied experiencing hearing difficulties presented $\mathrm{HL}$ when evaluated by audiometry. As hypoacusis is subjective in nature, this may be due to these individuals' failure to perceive their $\mathrm{HL}$, especially considering that mild $\mathrm{HL}$ was observed in 8 (72.7\%) of these cases.

Conductive HL was present in the majority of patients ( $60 \%$ ), while type-B curve was detected in $50 \%$ of patients, and the presence of fluid in the middle ear was identified in less than $8 \%$. These findings indicate that HL may result from the deposition of GAG in the middle ear, sequelae from prior otitis and/or ossicular injury. It is important to note that clinical ENT evaluations were not performed concomitantly with audiological testing, thus complicating any correlations made among otoscopic and audiometric findings. Previous studies have attributed conductive HL in MPS patients with the presence of effusion and ossicular injury in the middle ear. ${ }^{10}$ Other authors have suggested the likelihood of more than one etiological factor being involved, since ventilation tube placement in the TM has not been found to normalize HL. ${ }^{5,11}$

The presence of middle ear effusion was clinically verified in the minority of patients, which may be explained by an older patient age at the time of the initial evaluation (mean: 7.3 years), by TM thickening, ${ }^{12}$ which precludes the visualization of OME, or, alternatively, by the use of ERT.

To truly assess the impact of ERT, it would be necessary to compare the patients studied to a control group, which would obviously constitute reprehensible ethical conduct and prove difficult from a practical standpoint, since this condition is rare and assembling pairs among groups would be infeasible. Prospective phase-IV clinical trials have shown reduced sleep apnea in patients with MPS type I undergoing ERT, suggesting that this therapy has an effect on GAG deposition in the upper airways. ${ }^{8,13}$

In MPS type II, due to the specific type of inherent enzyme deficiency, HL appears to be mostly related to otosclerotic foci in the middle and inner ears. ${ }^{14}$ In an attempt to clarify the etiology of HL in patients with MPS, a previous study conducted histological evaluations of the temporal bone and found developmental interference at around 5 to 6 months of gestational age, as well as decreased mastoid pneumatization, the persistence of cartilaginous otic capsules adjacent to the posterior semicircular canal and persistence of the subarcuate artery. ${ }^{15}$

In addition, mixed $\mathrm{HL}$ appears to be a more common finding in patients with MPS. ${ }^{10,16}$ The elevated frequency of conductive HL detected herein may be due to a large number of patients with MPS VI in the present sample, since the sensorineural component is more strongly associated with MPS types I, II, and IV. ${ }^{16}$ When only patients with MPS type II were considered, the highest frequency of mixed HL was observed, thereby reinforcing this hypothesis. Another factor possibly associated with the observed elevated frequency of conductive HL may be a lack of surgical intervention in these patients, since ventilation tube placement can reduce the conductive component.

Although the etiology of sensorineural HL remains obscure, some authors have suggested that nerve compression by arachnoid hyperplasia and axonal destruction in the spiral ganglion may be responsible. ${ }^{15} \mathrm{~A}$ multicentric study of patients with MPS type II noted an increased frequency of sensorineural HL over time, at around $1 \mathrm{~dB}$ per year. ${ }^{9}$ No worsening of HL was observed in those patients with MPS type II who received two audiometric evaluations. This apparent stability might be due to selection bias, since audiometry is a subjective test, and patients with worsened cognition would not have a satisfactory response, leading to the selection of patients with a less severe phenotype on the subsequent audiometry exam. Accordingly, three patients with MPS type II underwent audiometry twice, yet two had no neurologic impairment. A lesser degree of hearing impairment has been described in patients with more attenuated forms of MPS type II. ${ }^{12}$ Another relevant factor to consider is that some interference in the progression of the sensorineural component may occur in patients who undergo ERT. Among the 8 patients with MPS type VI who were submitted to pure tone audiometry performed at 2 different time points, a worsening of auditory thresholds was observed in $35.5 \%$ of these cases, while worsening in the sensorineural component was seen in just 1 patient.

Auditory brainstem response measures of the electrophysiological threshold were found to be compatible with the auditory thresholds observed under audiometry. Only one patient presented delayed conduction in all waves with normal interpeaks. This finding is consistent with conductive $\mathrm{HL}^{17,18}$ as patients with this condition present severe bilateral mixed $\mathrm{HL}$, which explains the observed delay in nerve conduction. A previous study evaluating patients with MPS type II measured average hearing thresholds at $60 \mathrm{~dB}$ under $\mathrm{ABR},{ }^{12}$ which is consistent with our findings in patients with MPS II. Otoacoustic emission was not performed in these patients, as wheezing/loud breathing was found to interfere with this type of exam.

Initially, 24 patients were selected to participate in the present study, yet, during follow-up, only 15 were able to complete a subsequent audiological assessment. As most of these patients reside in the rural area, transportation options 
to the reference hospital located in the state capital (over $350 \mathrm{~km}$ away)are severely limited and often unavailable. Most of the patients who did not receive a follow-up evaluation had MPS type II, which may be associated with neurological impairment, thus presenting further difficulties with respect to transportation and caregiver assistance.

\section{Conclusion}

Patients with MPS present significant hearing impairment, with a prevalence approaching $100 \%$.

A low frequency of OME was seen in the evaluated patients, although an elevated incidence of conductive HL was found.

Although the impact of ERT on auditory symptoms remains unclear in MPS patients, otolaryngologists certainly play a crucial role in the multidisciplinary monitoring of these patients. Regular screening can provide early-stage auditory rehabilitation in these patients, thereby preventing $\mathrm{HL}$ from becoming an obstacle to their social integration.

\section{Conflict of Interests}

The authors have no conflict of interests to declare.

\section{References}

1 Muenzer J. Overview of the mucopolysaccharidoses. Rheumatology (Oxford) 2011;50(Suppl 5):v4-v12

2 Wraith JE. The mucopolysaccharidoses: a clinical review and guide to management. Arch Dis Child 1995;72(03):263-267

3 Baehner F, Schmiedeskamp C, Krummenauer F, et al. Cumulative incidence rates of the mucopolysaccharidoses in Germany. J Inherit Metab Dis 2005;28(06):1011-1017

4 Ternes D, Fernandes R, Mps R. Mucopolissacaridoses I, II e VI: Estudo epidemiológico comparativo entre as Regiões Nordeste (NE), Sudeste (SE) e Sul (S) do Brasil. XVI Encontro de Geneticistas do Rio Grande do Sul, Porto Alegre. 2008. Accessed 26/11/2014

5 Wold SM, Derkay CS, Darrow DH, Proud V. Role of the pediatric otolaryngologist in diagnosis and management of children with mucopolysaccharidoses. Int J Pediatr Otorhinolaryngol 2010;74 (01):27-31
6 Pastores GM, Arn P, Beck M, et al. The MPS I registry: design, methodology, and early findings of a global disease registry for monitoring patients with Mucopolysaccharidosis Type I. Mol Genet Metab 2007;91(01):37-47

7 Hendriksz CJ, Giugliani R, Harmatz P, et al. Multi-domain impact of elosufase alfa in Morquio A syndrome in the pivotal phase III trial. Mol Genet Metab. 2015;114(02):178-185. doi:10.1016/j. ymgme.2014.08.012

8 Wraith JE, Clarke LA, Beck M, et al. Enzyme replacement therapy for mucopolysaccharidosis I: a randomized, double-blinded, placebo-controlled, multinational study of recombinant human alpha-L-iduronidase (laronidase). J Pediatr 2004;144(05):581588

9 Keilmann A, Nakarat T, Bruce IA, Molter D, Malm G; HOS Investigators. Hearing loss in patients with mucopolysaccharidosis II: data from HOS - the Hunter Outcome Survey. J Inherit Metab Dis 2012;35(02):343-353

10 Papsin BC, Vellodi A, Bailey CM, Ratcliffe PC, Leighton SE. Otologic and laryngologic manifestations of mucopolysaccharidoses after bone marrow transplantation. Otolaryngol Head Neck Surg 1998; 118(01):30-36

11 Motamed M, Thorne S, Narula A. Treatment of otitis media with effusion in children with mucopolysaccharidoses. Int J Pediatr Otorhinolaryngol 2000;53(02):121-124

12 Cho Y-S, Kim JH, Kim TW, Chung SC, Chang S-A, Jin D-K. Otologic manifestations of Hunter syndrome and their relationship with speech development. Audiol Neurotol 2008;13 (03):206-212

13 Giugliani R, Federhen A, Rojas MV, et al. Mucopolysaccharidosis I, II, and VI: Brief review and guidelines for treatment. Genet Mol Biol 2010;33(04):589-604

14 Zechner G, Moser M. Otosclerosis and mucopolysaccharidosis. Acta Otolaryngol 1987;103(5-6):384-386

15 Hayes E, Babin R, Platz C. The otologic manifestations of mucopolysaccharidoses. Am J Otol 1980;2(02):65-69

16 Simmons MA, Bruce IA, Penney S, Wraith E, Rothera MP. Otorhinolaryngological manifestations of the mucopolysaccharidoses. Int J Pediatr Otorhinolaryngol 2005;69(05):589-595

17 Bento RF. Tratado de Otologia [Internet]. EdUSP; 1998 [cited 2014 Dec 8]. Available from: http://books.google.com/books?id=vld9oYv_E-4C\&pgis=1

18 Gelfand SA. Essentials of Audiology [Internet]. Thieme; 2011 [cited 2014 Dec 8]. Available from: http://books.google.com/books?id= SGdR8uCuRHUC\&pgis $=1$ 